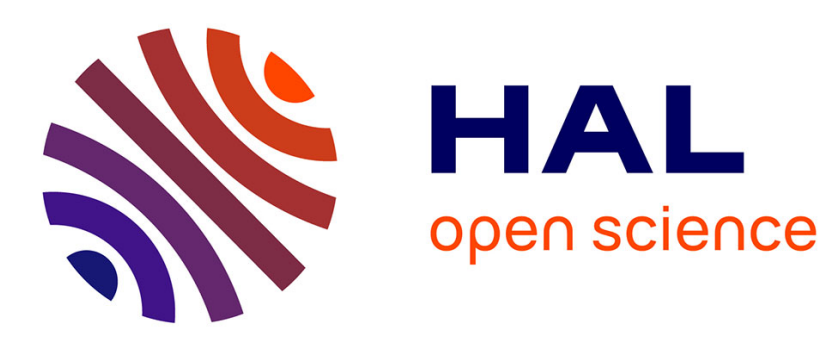

\title{
Polarization selectivity in vibrational electron-energy-loss spectroscopy
}

G. Radtke, D. Taverna, N. Menguy, S. Pandolfi, A. Courac, Y. Le Godec, O. 1. Krivanek, T. c. Lovejoy

\section{- To cite this version:}

G. Radtke, D. Taverna, N. Menguy, S. Pandolfi, A. Courac, et al.. Polarization selectivity in vibrational electron-energy-loss spectroscopy. Physical Review Letters, 2019, 123 (25), pp.256001. 10.1103/PhysRevLett.123.256001 . hal-02434893

\section{HAL Id: hal-02434893 https://hal.sorbonne-universite.fr/hal-02434893}

Submitted on 10 Jan 2020

HAL is a multi-disciplinary open access archive for the deposit and dissemination of scientific research documents, whether they are published or not. The documents may come from teaching and research institutions in France or abroad, or from public or private research centers.
L'archive ouverte pluridisciplinaire HAL, est destinée au dépôt et à la diffusion de documents scientifiques de niveau recherche, publiés ou non, émanant des établissements d'enseignement et de recherche français ou étrangers, des laboratoires publics ou privés. 


\title{
Polarization selectivity in vibrational electron-energy-loss spectroscopy
}

\author{
G. Radtke, ${ }^{1, *}$ D. Taverna,${ }^{1}$ N. Menguy,${ }^{1}$ S. Pandolfi, ${ }^{1}$ A. Courac, ${ }^{1}$ Y. Le Godec,${ }^{1}$ O. L. Krivanek, ${ }^{2}$ and T. C. Lovejoy ${ }^{2}$ \\ ${ }^{1}$ Sorbonne Université, Muséum National d'Histoire Naturelle, \\ UMR CNRS 7590, IRD, Institut de Minéralogie, \\ de Physique des Matériaux et de Cosmochimie, IMPMC, 75005 Paris, France \\ ${ }^{2}$ Nion Co., 11511 NE 118th St., Kirkland WA 98034, USA
}

\begin{abstract}
Orientation-dependent aloof-beam vibrational electron-energy-loss spectroscopy is carried out on uniaxial icosahedral $\mathrm{B}_{12} \mathrm{P}_{2}$ submicron crystals. We demonstrate that the high sensitivity of the signal to the crystal orientation allows for an unambiguous determination of the symmetry of normal-modes occurring at the Brillouin zone center of this anisotropic compound. The experimental results are assessed using first-principles quantum mechanical calculations (density functional theory) of the dielectric response of the specimen. The high spatial resolution inherent to this technique when implemented in the transmission electron microscope thus opens the door to nanoscale orientationdependent vibrational spectroscopy.
\end{abstract}

PACS numbers: 79.20.Uv, 68.37.Ma, 78.20.Bh

Ultra-high resolution electron-energy-loss spectroscopy (EELS) in the (scanning) transmission electron microscope ((S)TEM) has recently attracted a considerable attention as an emerging technique to investigate the vibrational properties of condensed matter with an unprecedented spatial resolution [1-3]. When implemented in a non-penetrating geometry, i.e. using an electron beam positioned at a finite distance away from the edge of the sample, energy loss processes are dominated by dipole scattering arising from long-range electric fields generated through lattice or molecular vibrations of polar specimens. This aloof geometry has recently proved very effective for imaging confined surface phonon modes in nanostructures $[2,4]$ and highly beneficial for studying vibrational properties of beam-sensitive specimens [5-7] due to the drastic reduction of damage caused by the aloof electron beam when compared to more standard transmission geometries [8].

In this Letter, we add a new dimension to this approach by demonstrating that it can also be employed to assess the symmetry of normal modes occuring at the Brillouin zone center of anisotropic materials. Indeed, although the electron probe lacks the intrinsic angular selectivity of polarized photons used in optical spectroscopies, the scattering geometry in EELS provides a similar insight into the symmetry of vibrational excitations with the further advantage of a high spatial resolution. This is illustrated through the study of uniaxial icosahedral boron subphosphide $\mathrm{B}_{12} \mathrm{P}_{2}$ where phonon mode symmetry is unambiguously retrieved from spectra acquired on crystals with distinct orientations.

$\mathrm{B}_{12} \mathrm{P}_{2}$ is a member of the $\alpha$-rhombohedral boron structure group that includes compounds such as $\alpha-\mathrm{B}_{12}$, $\mathrm{B}_{12} \mathrm{As}_{2}, \mathrm{~B}_{12} \mathrm{O}_{2}$ or $\mathrm{B}_{12} \mathrm{C}_{3}$ and, as most of them, displays a number of technologically interesting properties. $\mathrm{B}_{12} \mathrm{P}_{2}$ is a wide band gap semiconductor $(3.35 \mathrm{eV})[9]$, a thermoelectric with a high positive Seebeck coefficient [10] and displays both high thermal and chemical stabilities
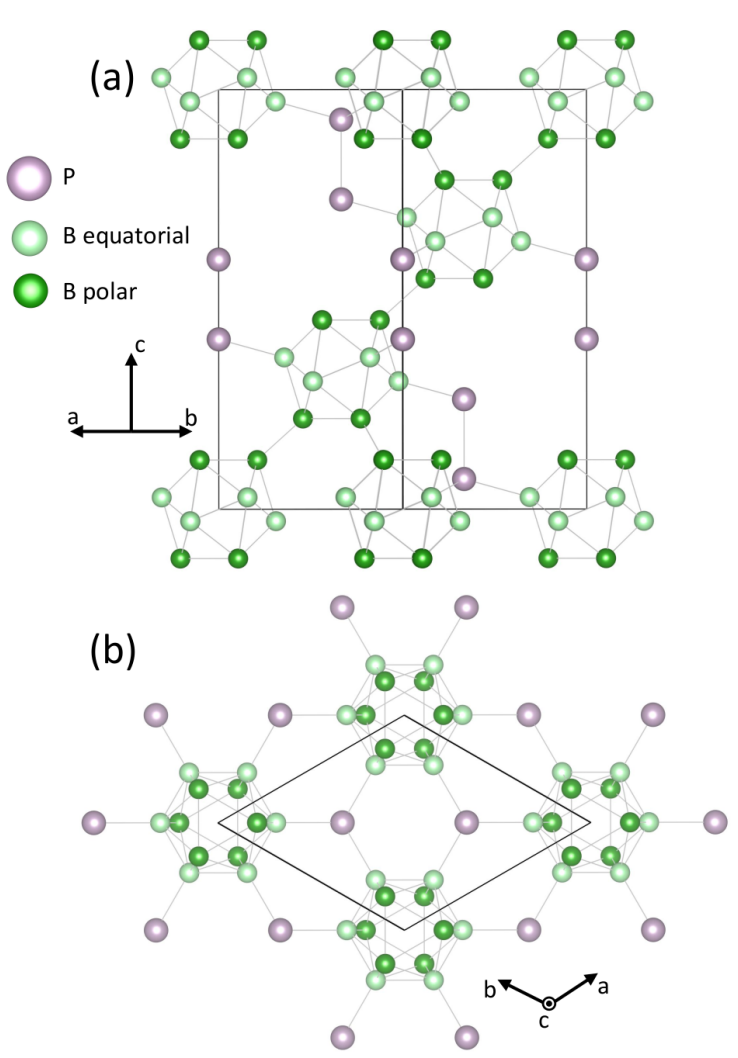

FIG. 1: (color online) Structure of $\mathrm{B}_{12} \mathrm{P}_{2}$ : (a) side-view and (b) top view of the hexagonal triple cell. For clarity, parts of the lattice have been omitted in (b). Phosphorus atom are in light purple, polar boron in deep green and equatorial boron in light green.

as well as an extreme resistance to damage induced by high energy particle radiations $[11,12]$. This peculiar behaviour is attributed to a self-healing mechanism whose origin has recently been proposed to lie in the very low activation energy for the diffusion and recombination of 
interstitial-vacancy pairs [13] and makes it a potential candidate for betavoltaics [12].

This compound crystallizes in the $R \overline{3} \mathrm{~m}$ rhombohedral space group. Its atomic structure is represented in Fig. 1 within the hexagonal triple cell. It consists in $\mathrm{B}_{12}$ icosahedra linked together either through B-B bonds along the $c$-axis of the crystal or through B-P-B bonds in the perpendicular plane. Two crystallographically inequivalent boron sites are therefore present in the structure. The polar site, represented with dark green atoms in Fig. 1, are located on six vertices of the 12-atom cluster to form the top and bottom triangular faces and are exclusively bonded to boron atoms, belonging either to the same or to an adjacent icosahedron. The equatorial sites, represented by light green atoms, form a puckered hexagon in the plane perpendicular to the $c$-axis. As shown in Fig. 1, phosphorus cations are fourfold coordinated, forming bonds with an other cation and with three adjacent equatorial boron atoms.

As for its symmetry, $\mathrm{B}_{12} \mathrm{P}_{2}$ is an uniaxial material, leading to EELS active modes with well-defined characteristics at the $\Gamma$ point. The decomposition of the vibrational degrees of freedom on the irreducible representations of the crystal point group $\left(D_{3 d}\right)$ gives [16]

$$
\Gamma_{\text {vib. }}=5 A_{1 g} \oplus 2 A_{1 u} \oplus 2 A_{2 g} \oplus 5 A_{2 u} \oplus 7 E_{u} \oplus 7 E_{g},
$$

from which the $A_{2 u} \oplus E_{u}$ acoustic modes should be removed to obtain the symmetry properties of the 39 remaining optical modes

$$
\Gamma_{\text {op. }}=5 A_{1 g} \oplus 2 A_{1 u} \oplus 2 A_{2 g} \oplus 4 A_{2 u} \oplus 6 E_{u} \oplus 7 E_{g} .
$$

As EELS active modes in aloof geometry are necessarily those generating a non-zero electric polarization, we can conclude that a maximum of ten distinct peaks can be observed on the experimental spectra, four of $A_{2 u}$ and six of $E_{u}$ symmetry, the former giving rise to a macroscopic polarization parallel to the $c$-axis and the latter, to a polarization in the perpendicular plane.

The structure and vibrational properties of $\mathrm{B}_{12} \mathrm{P}_{2}$ have been determined in the framework of density functional theory (DFT) using the QUANTUM ESPRESSO [17] package. Calculations were performed in the 14-atom trigonal unit cell using ultrasoft pseudopotentials [18] with plane-wave and charge-density cutoffs of $80 \mathrm{Ry}$ and $640 \mathrm{Ry}$, respectively. The Brillouin zone of the trigonal crystal was sampled using a $8 \times 8 \times 8 \mathrm{k}$-point MonkhorstPack grid [19] and the generalized gradient approximation of Perdew, Burke, and Ernzerhof [20] (GGA-PBE) employed for exchange and correlation. This choice has been guided by the fairly accurate results usually provided by GGAs on the structural properties of strongly bound solids [14] and confirmed a posteriori by the excellent agreement obtained between optimized lattice parameters of $a=5.990$ and $c=11.845 \AA$ (hexagonal triple cell) and the average experimental values of

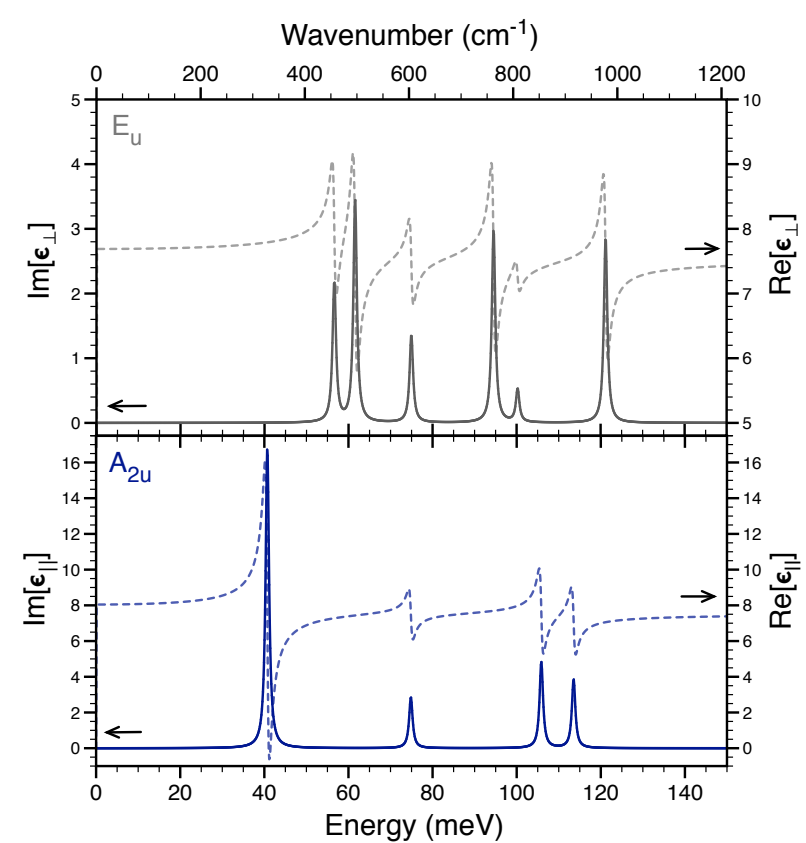

FIG. 2: (color online) Principal components of the local dielectric tensor $\overleftrightarrow{\epsilon}(\omega)$ of $\mathrm{B}_{12} \mathrm{P}_{2}$ in the infrared domain of frequencies calculated with a damping parameter $\Gamma_{m}=4 \mathrm{~cm}^{-1}$. Component perpendicular $\left(\epsilon_{\perp}\right)$ and parallel $\left(\epsilon_{||}\right)$to the $c$-axis of the crystal are shown in the lower and upper panels, respectively. Real and imaginary parts of the dielectric tensor components are represented, respectively, with dashed and solid lines.

5.986 and $11.848 \AA$ reported in Ref. 15 for stoichiometric $\mathrm{B}_{12} \mathrm{P}_{2}$. The dynamical matrix at the $\Gamma$ point, electronic dielectric and Born effective charge tensors obtained using density functional perturbation theory (DFPT) were employed to calculate the principal components of the low-frequency local $(\mathbf{q}=0)$ dielectric tensor $\overleftrightarrow{\epsilon}(\omega)$ shown in Fig. 2, entirely from first-principles [21, 22]. Throughout this study, an average atomic mass of 10.811 a.m.u. was employed for boron, accounting for the relative natural abundance of ${ }^{10} \mathrm{~B}$ and ${ }^{11} \mathrm{~B}$ isotopes [24]. Consistently with our previous group-theoretical analysis, $\epsilon_{\|}(\omega)$ and $\epsilon_{\perp}(\omega)$, where the parallel and perpendicular subscripts refer to the $c$-axis of the hexagonal triple cell, are respectively built from four resonances associated with the four modes of $A_{2 u}$ symmetry and six resonances associated with the six modes of $E_{u}$ symmetry occuring at the Brillouin zone center of $\mathrm{B}_{12} \mathrm{P}_{2}$. A schematic representation of these modes is provided in the Supplemental Material [23]. Note that these modes could be distinguished in polarized infrared absorption (IR) experiments carried out on single crystals as the former are active when light is polarized parallel to the $c$-axis and the latter when light is polarized in the perpendicular plane.

The local dielectric tensor is the key component entering the classical description of electron scattering by sur- 

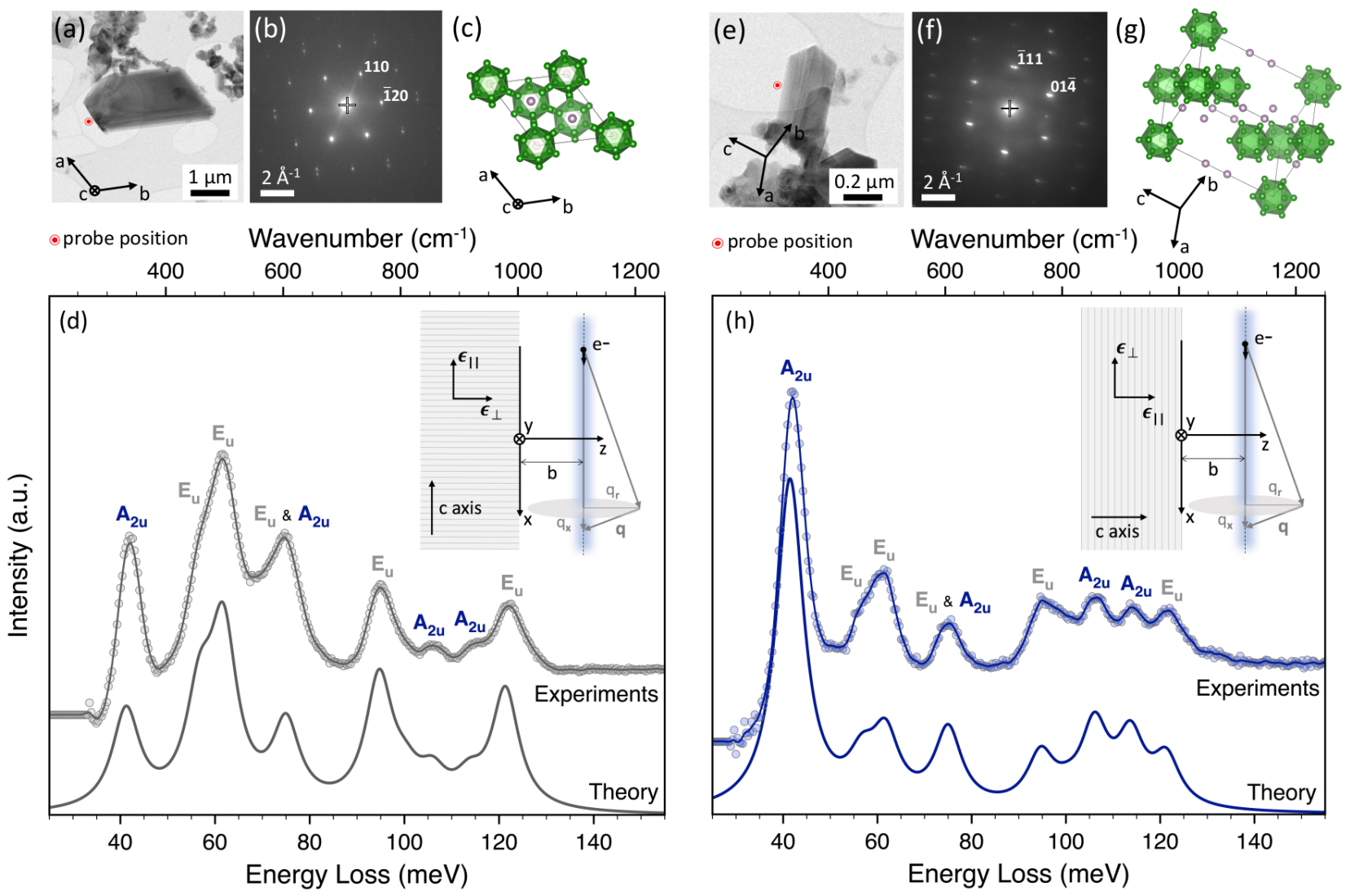

FIG. 3: (color online) Comparison between the experimental and theoretical vibrational EELS spectra of $\mathrm{B}_{12} \mathrm{P}_{2}$ for two microcrystals with different crystallographic orientations. (a) Conventional bright-field TEM image of the crystal acquired at $200 \mathrm{keV}$ prior to the STEM experiment. The approximate beam position used for the $30 \mathrm{keV}$ STEM-EELS experiment is indicated with a red point. (b) Indexed $30 \mathrm{keV}$ diffraction pattern acquired in the STEM just before the EELS experiment showing that the electron beam propagates parallel to the $c$-axis of the crystal. (c) Corresponding orientation of the hexagonal triple crystal cell. (d) Comparison between the experimental EELS spectrum acquired in aloof geometry at a distance $\mathrm{b} \approx 10 \mathrm{~nm}$ from the specimen edge and the theoretical spectrum calculated using Eq. 1 and the principal components of the dielectric tensor shown in Fig. 2. The symmetry of the modes corresponding to each peak is indicated. The idealized geometry employed in the calculations is shown in inset. (e)-(h) Same as (a)-(d) with the c-axis of the microcrystal close to perpendicular to the electron beam.

face phonons occurring in aloof geometry in the context of the local continuum dielectric model $[4,25]$. In this classical, quasi-static approach [25-28], the energy-loss probability per unit angular frequency and path length, for an electron travelling along a rectilinear trajectory parallel to, and at a finite distance $b$ from, the surface of a specimen occupying a half-space, is given by

$$
\begin{aligned}
\frac{\mathrm{d}^{2} P(\omega, \mathrm{b})}{\mathrm{d} \omega \mathrm{d} x} & =\frac{e^{2}}{(2 \pi)^{2} v^{2} \epsilon_{0} \hbar} \times \\
& \int_{-\infty}^{\infty} \operatorname{Im}\left[\alpha\left(\omega, q_{x}, q_{y}\right)\right] \frac{e^{-2 \mathrm{~b} \sqrt{q_{x}^{2}+q_{y}^{2}}}}{\sqrt{q_{x}^{2}+q_{y}^{2}}} \mathrm{~d} q_{y},
\end{aligned}
$$

where $v$ the electron velocity and $\left(q_{x}, q_{y}\right)$ the components of the scattering vector in the surface plane (see the scattering geometry in the insets of Fig. 3 (d) and (h) for the orientation of the cartesian coordinates). This expression involves the imaginary part of the polarizability of the specimen, which maxima correspond to the energy of the surface vibrational excitations. When both the surface and the electron beam are parallel to the $c$-axis, the polarizability can be expressed as

$$
\alpha(\omega)=\frac{\sqrt{\epsilon_{\perp}(\omega)\left(q_{x}^{2} \epsilon_{\|}(\omega)+q_{y}^{2} \epsilon_{\perp}(\omega)\right) / Q^{2}}-1}{\sqrt{\epsilon_{\perp}(\omega)\left(q_{x}^{2} \epsilon_{\|}(\omega)+q_{y}^{2} \epsilon_{\perp}(\omega)\right) / Q^{2}}+1} .
$$

In this expression, $Q=\sqrt{q_{x}^{2}+q_{y}^{2}}$ is the norm of the scattering vector parallel to the surface [25]. It should be stressed here that Eq. 2 depends explicitly on the scattering vector, leading to an imbalance in the role played by the two dielectric components. On the contrary, for 
a surface perpendicular to the $c$-axis of the crystal, the expression of the polarizability is particularly simple as it becomes independent of the scattering vector

$$
\alpha(\omega)=\frac{\sqrt{\epsilon_{\|}(\omega) \epsilon_{\perp}(\omega)}-1}{\sqrt{\epsilon_{\|}(\omega) \epsilon_{\perp}(\omega)}+1},
$$

and only involves the geometrical mean of the two dielectric components [29].

In expressions (1) and (2), the scattering vector along the electron trajectory is given by $q_{x}=\omega / v$ which, for a typical loss of $70 \mathrm{meV}$ and a primary electron energy of $30 \mathrm{keV}$, is of the order of $10^{-4} \AA^{-1}$. The amplitude of the perpendicular component $\left(q_{r}\right.$ in insets of Fig. 3 (d) and (h)) is more difficult to assess as it is usually not limited by the relatively large collection angle used in the experiments, but most likely by the low- $q$ filter term occurring in the integral of Eq. 1. A typical average value up to an order of magnitude larger than the parallel component can however be estimated based on Eq. 1, resulting in a scattering vector oriented predominantly in the plane perpendicular to the beam trajectory.

Anisotropy resulting from the differences between Eq. 2 and Eq. 3 was mesured experimentally on $\mathrm{B}_{12} \mathrm{P}_{2}$ microcrystals with different orientations. Polycrystalline $\mathrm{B}_{12} \mathrm{P}_{2}$, synthetized by ultra-fast mechanochemical method through a reaction between $\mathrm{BPO}_{4}, \mathrm{Mg}$ and $\mathrm{MgB}_{2}$ [31], was crushed into powder and dispersed on a lacey carbon grid. A first TEM inspection of the specimen led to the identification of micron or submicron crystals with sharp edges located in large carbon film holes and distinct crystallographic orientations, as shown in Fig. 3(a) and (e). The specimen was then analyzed in a Nion U-HERMES 200 system operated at 30kV. Diffraction patterns recorded prior to the EELS acquisition and shown in Fig. 3(b) and (f) illustrate the geometry of these experiments: for the crystal shown in (a), the $c$-axis is parallel to the electron beam and parallel to the surface whereas for the crystal shown in (e), the $c$-axis is approximately perpendicular to the electron beam and to the surface. Fig. 3(c) and (g) summarize these results by showing the hexagonal triple cell viewed along the beam direction.

Vibrational EELS spectra were acquired in aloof geometry at a distance of $10 \mathrm{~nm}$ from the edge of the specimen. The final spectra shown in Fig. 3(d) and (h) result from the acquisition of 5000 spectra of $50 \mathrm{~ms}$ each, subsequently realigned and summed, leading to an overall energy resolution of $6 \mathrm{meV}$. Convergence and collection semi-angles were set to $\alpha=\beta=10 \mathrm{mrad}$. In both cases, a total number of eight peaks [30], including the low intensity shoulder of the peak located at $62 \mathrm{meV}$, is observed in the $40-125 \mathrm{meV}$ energy range. The essential difference between the two spectra arises from the dramatic variations of the peak intensities induced by the crystal orientation.
In order to gain insight into the origin of these variations, theoretical spectra were calculated using Eq. 1, the dielectric components shown in Fig. 2 and the polarizabilities given in Eq. 2 and (3). These last two expressions correspond to the simplified geometries shown in inset of Fig. 3(d) and (h). Although significant deviations from these idealized geometries are observed in actual experiments, the predictions provided by the local continuum dielectric model reproduce very well the experimental results for both orientations. These results allow for precise symmetry assignment of each visible peak and lead to an important observation: whereas the intensity of the peaks associated with phonon modes of $E_{u}$ symmetry is largely enhanced in the first orientation (see Fig. 3(d)), it is strongly reduced in the second (see Fig. 3(h)). Conversely, the intensity of the peaks corresponding to phonons of $A_{2 u}$ symmetry is relatively weak in the first orientation but reinforced in the second. This behaviour is a direct consequence of Eq. 2 where the larger perpendicular $\left(q_{y}\right)$ component of the scattering vector strengthens the intensity of modes generating an in-plane polarization compared to the geometrical average of Eq. 3. The high sensitivity of the aloof-beam EELS signal to the crystal orientation therefore appears as a very efficient means to discriminate phonon modes according to the direction of the macroscopic polarization they are building, either parallel or perpendicular to the crystal $c$-axis. This crystal orientation dependence can thus be employed to determine the symmetry of EELS active normal modes. Note that although similar information can be retrieved from polarized IR absorption experiments, the lack of sufficiently large single crystals, however, often hinders such analysis $[32,33]$.

In conclusion, the present work demonstrates the capability of aloof-beam vibrational EELS to access the symmetry of phonon modes observed in anisotropic crystals. Distinct intensity variations are observed for peaks associated with phonons modes building macroscopic polarizations with different spatial orientations when changing the geometry of the experiment, i.e. changing the relative orientation of the electron beam and the surface with respect to the crystal axes. A great advantage of this approach resides in the high spatial resolution of EELS when implemented in a (S)TEM, therefore allowing to carry out this type of analysis at the nanoscale.

This work was granted access to the HPC resources of IDRIS under the allocation 2019-A0060910820 attributed by GENCI (Grand Equipement National de Calcul Intensif). The authors thank V.A. Mukhanov for the sample synthesis as well as K. March, E. Balan and P. Giura for very fruitful discussions. This work was supported by Innovation Program Satt IDF Innov. (grant agreement $\left.\mathrm{N}^{\circ} \mathrm{L} 14 \mathrm{~S} 495\right)$. 
* Electronic address: sorbonne-universite.fr

[1] O.L. Krivanek T.C. Lovejoy, N. Dellby, T. Aoki, R.W Carpenter, P. Rez, E. Soignard, J. Zhu, P.E. Batson, M.J. Lagos, R.F. Egerton and P.A. Crozier Nature 514, 209 (2014).

[2] M. J. Lagos, A. Trügler, U. Hohenester and P.E. Batson Nature 543, 529 (2017).

[3] F.S. Hage, D.M. Kepaptsoglou, Q.M. Ramasse and L.J. Allen Phys. Rev. Lett. 122, 016103 (2019).

[4] H. Lourenço-Martins and M. Kociak Phys. Rev. X 7, 041059 (2017).

[5] P. Rez, T. Aoki, K. March, D. Gur, O.L. Krivanek, N. Dellby, T.C. Lovejoy, S.G. Wolf and H. Cohen Nat. Commun. 7, 10945 (2016).

[6] J. A. Hachtel, J. Huang, I. Popovs, S. Jansone-Popova, J. K. Keum, J. Jakowski, T. C. Lovejoy, N. Dellby, O.L. Krivanek, J.C. Idrobo Science 363, 525 (2019).

[7] P. Crozier T. Aoki and Q. Liu Ultramic. 16930 (2016).

[8] R.F. Egerton Ultramic. 15995 (2015).

[9] G.A. Slack, T.F. McNelly and E.A. Taft J. Phys. Chem. Solids 44, 1009 (1983).

[10] Y. Kumashiro, T. Yokoyama, K. Sato, and Y. Ando J. Solid State Chem. 154, 33 (2000).

[11] M. Carrard, D. Emin and L. Zuppiroli Phys. Rev. B 51, 11270 (1995).

[12] D. Emin J. Solid State Chem. 179, 2791 (2006)

[13] S. P. Huber, E. Gullikson, C. D. Frye, J. H. Edgar, R. W. E. van de Kruijs, F. Bijkerk, and D. Prendergast Chem. Mater. 28, 8415 (2016).

[14] F. Tran, J. Stelzl, and P. Blaha J. Chem. Phys. 144, 204120 (2016).

[15] G.A. Slack, K.E. Morgan J. Phys. Chem. Solids 75, 1054 (2014).

[16] P.M.A. Sherwood Vibrational spectroscopy of solids, Cambridge University Press (1972).

[17] P. Giannozzi et al. J. Phys. : Condens. Matter 21, 395502 (2009).

[18] K.F. Garrity, J.W. Bennett, K.M. Rabe and D. Vander- bilt Comp. Mater. Sci. 81, 446 (2014).

[19] H.J. Monkhorst and J.D. Pack Phys. Rev. B 13, 5188 (1976).

[20] J.P. Perdew, K. Burke and M. Ernzerhof Phys. Rev. Lett. 77, 3865 (1996)

[21] X. Gonze and C. Lee Phys. Rev. B 55, 10355 (1997).

[22] S. Baroni, S. de Gironcoli, A. Dal Corso and P. Giannozzi Rev. Mod. Phys. 73, 515 (2001).

[23] See Supplemental Material at http://XXX for a schematic representation of the atomic displacements associated with the modes contributing to the dielectric tensor.

[24] Although previous studies (see for example Refs. [32, 33]) on IR absorption in $\mathrm{B}_{12} \mathrm{P}_{2}$ report the presence of split phonon bands built from a strong ${ }^{11} \mathrm{~B}$ line and a weaker ${ }^{10} \mathrm{~B}$ shoulder at higher energy, they were not distinguishable in the present experiments due to our limited energy resolution. We therefore only considered an averaged boron atomic mass in the calculations.

[25] G. Radtke, D. Taverna, M. Lazzeri and E. Balan, Phys. Rev. Lett. 119, 027402 (2017).

[26] P. Batson Ultramic. 11299 (1983).

[27] A. Howie Ultramic. 11141 (1983).

[28] A.A. Govyadinov, A. Konečná, A. Chuvilin, S. Vélez, I. Dolado, A.Y. Nikitin, S. Lopatin, F. Casanova, L.E. Hueso, J. Aizpurua and R. Hillenbrand Nat. Comm. 9 95 (2017).

[29] A.A. Lucas and J.P. Vigneron Solid State Comm. 49327 (1984)

[30] Only eight peaks are visible on the experimental spectra out of a total of ten predicted EELS active modes. This is due to both, an overlap of two modes of $A_{2 u}$ and $E_{u}$ symmetries occurring around $75 \mathrm{meV}$ (see Fig. 2) and to the low-intensity of the $E_{u}$ mode at $100 \mathrm{meV}$ which is not resolved with our energy resolution.

[31] V.A. Mukhanov, D. Vrel, P.SP Sokolov, Y. Le Godec and V. Solozhenko Dalton Trans. 4510122 (2016).

[32] H.J. Becher and F.Thévenot Z. anorg. allg. Chem. 410 274 (1974).

[33] H. Werheit, U. Kuhlmanna, K. Shiraib, Y. Kumashiro J. Alloys Comp. 233121 (1992). 